Original research article

\title{
Improved prediction of CPAP failure using T90, age and gender
}

\author{
David Slouka ${ }^{1 *}$, Monika Honnerova ${ }^{2}$, Petr Hosek ${ }^{3}$, Bretislav Gal ${ }^{4}$, Ondrej Trcka ${ }^{1}$, Tomas Kostlivy ${ }^{1}$, \\ Jana Landsmanova ${ }^{5}$, David Havel ${ }^{2}$, Martina Baneckova ${ }^{6}$, Radek Kucera ${ }^{7}$ \\ ${ }^{1}$ Charles University, Faculty of Medicine in Pilsen, Ear, Nose and Throat Department, Pilsen, Czech Republic \\ ${ }^{2}$ Charles University, Faculty of Medicine in Pilsen, Department of Pneumology, Pilsen, Czech Republic \\ ${ }^{3}$ Charles University, Faculty of Medicine in Pilsen, Biomedical Center, Pilsen, Czech Republic \\ ${ }^{4}$ Masaryk University, Faculty of Medicine, Ear, Nose and Throat Department, Brno, Czech Republic \\ ${ }^{5}$ Charles University, Faculty of Medicine in Pilsen, Department of Obstetrics and Gynaecology, Pilsen, Czech Republic \\ ${ }^{6}$ Charles University, Faculty of Medicine in Pilsen, Department of Pathology, Pilsen, Czech Republic \\ ${ }^{7}$ Charles University, Faculty of Medicine in Pilsen, Department of Nuclear Medicine Laboratory of Immunoanalysis, Pilsen, Czech Republic
}

\begin{abstract}
Sleep apnea syndrome is associated with increased risk of cardiovascular disease. In treating older patients, there is a special emphasis put on minimally invasive and conservative procedures and a simple method for predicting the potential for treatment success is essential. Continuous positive airway pressure (CPAP) is the first choice for treatment, however, it is not always successful. In cases where CPAP was unsuccessful, treatment with bilevel positive airway pressure (BiPAP) is the next treatment option. In this study, we examine commonly evaluated respiratory parameters, obesity, and age relative to their ability to predict CPAP failure. We also tried to find differences in the predictive ability of these parameters in older and younger patients. The predictive ability, relative to CPAP failure, was examined for each individual parameter as well as for combinations of parameters. All variables had a statistical association with CPAP failure; failure prediction reliability ranged from poor to moderate. Combining T90, age, and gender can be used to find patients who will benefit from $\mathrm{BiPAP}$ as the first choice for treatment. An initial BiPAP indication can produce relevant reductions in treatment cost.
\end{abstract}

Keywords: Age; CPAP failure; Obesity; Prediction of failure; Sleep apnea syndrome

\section{Introduction}

Sleep-disordered breathing affects approximately $15 \%$ of the population (Mannarino et al., 2012). According to epidemiological studies, more than half of such cases are sleep apnea syndrome (SAS), which affects approximately $2 \%$ of middle-aged women and $4 \%$ of middle-aged men.

Sleep breathing disorders in the elderly can be accompanied by severe cardiovascular complications (Monahan and Redline, 2011). SAS may cause arterial hypertension (Tureto-Borges et al., 2018); the presence of apneic episodes disrupts the physiological decrease in systolic and diastolic blood pressure (non-dipper) that normally occurs during night-time sleep. Sleep apnea syndrome is also a risk factor for adverse cardiovascular events such as myocardial infarction, heart failure, and stroke, all of which occur more frequently in SAS patients (Barbé et al., 2012; Kasai, 2012). In patients with untreated SAS, atrial fibrillation is more common and reoccurs more frequently even after successful cardioversion. Similarly, in diabetic patients, glycemic control can be more difficult to achieve without SAS treatment due to changes leading to insulin resistance. Central nervous system symptoms, with excessive daytime sleepiness being the main clinical symptom (Dostálová et al., 2012; Tóthová et al., 2015), severely limit everyday life activities and, in some cases, leads to disability. In older SAS patients especially, associated memory problems and depression can further worsen the quality of life (Bruin and Bagnato, 2010; Onen and Onen, 2010).

Etiologically, there are three forms of SAS: obstructive (OSAS), central, and mixed SAS of different severity. According to the apnea-hypopnea index (AHI) SAS can be classified as AHI 0-4.9, without SAS, AHI 5-14.9, mild SAS, AHI 15-29.9, moderate SAS, and AHI $\geq 30$, severe SAS (Butner et al., 2013; Epstein et al., 2009). With increasing age, the proportion of the obstructive form of SAS (OSAS) increases, accounting for up to $80 \%$ of senior SAS cases. Central and mixed forms of SAS represent about 10\% each (Monahan and Redline, 2011). The first steps in the SAS diagnostic process include sleep monitoring in the Somnology Department and an ear-nose-throat examination (ENT) of the upper respiratory tract. Then, if necessary, specialists from internal medicine, psychology, and stomatology are asked to participate in the diagnosis and/or treatment (Vozoris, 2012).

\footnotetext{
* Author for correspondence: David Slouka, Charles University, Faculty of Medicine in Pilsen, Ear, Nose and Throat Department, Edvarda Beneše 13, 30599 Pilsen, Czech Republic; e-mail: slouka@fnplzen.cz http://doi.org/10.32725/jab.2018.008

Submitted: 2018-10-09 • Accepted: 2018-12-20 • Prepublished online: 2019-01-10

J Appl Biomed 17/1: 76-81 • EISSN 1214-0287 • ISSN 1214-021X

(c) 2018 The Authors. Published by University of South Bohemia in České Budějovice, Faculty of Health and Social Sciences.

This is an open access article under the CC BY-NC-ND license.
} 
For patients with moderate to severe SAS, positive airway pressure (PAP) therapy is primarily indicated. The first treatment choice is a single-level device with continuous positive airway pressure CPAP (Chowdury et al., 2012). It is applied through the nose or a full face mask (Williams et al., 2013). Before treatment, it is necessary to carefully choose the mask and determine the correct therapeutic pressure needed to prevent all adverse breathing events (Leidag et al., 2008). If CPAP is unsuccessful, bi-level positive airway pressure (BiPAP) is the next treatment step. Surgical treatment is an option depending on situations where the effect of PAP is limited by the upper airway obstruction (Ephros et al., 2010; Maurer, 2010) or when conservative therapy for severe OSAS has failed (Browaldh et al., 2009; Jalbert et al., 2012).

For older patients, CPAP treatment can initially be technically complicated and difficult to understand. Even a small leak around the nose or full face mask can cause bad function and CPAP failure (Leidag et al., 2008).

Approximately $13 \%$ of patients with OSAS experience CPAP treatment failure (Mulgrew et al., 2010; Schäfer et al., 1998). In these patients, CPAP prevents apneic episodes but does not resolve the problem of hypoventilation. Residual AHI values may be normal, but hyposaturation persists. The risk of hyposaturation (hypoventilation) may be particularly high in older OSAS patients. To date, there are no studies that compared the success rates of PAP-treatment in older and younger patients with OSAS and few studies that evaluated factors capable of predicting successful CPAP treatment (Schäfer et al., 1998; Sopkova et al., 2009).

The aim of our study was to compare selected characteristics of SAS in two groups of patients: younger adults $(<64.9$ years) and older patients ( $>65$ years). Additionally, we wanted to differentiate patients most likely to benefit from CPAP treatment from those who needed BiPAP as the initial treatment (Dohi et al., 2008; Piper et al., 2008).

\section{Materials and methods}

\section{Materials}

From 2005-2015 a total of 1,253 patients were treated for SAS at the University Hospital in Pilsen, Czech Republic. The study sample consisted of 665 consecutive patients (478 males (72\%) and 187 females (28\%) with a mean age of $59.1 \pm 9.7$ years) who met the inclusion criteria (Table 1). A retrospective analysis of the patient's data was carried out. The Ethics Committee of the University Hospital in Pilsen granted approval for data analyses.

\section{Methods}

This work was a single center, retrospective cohort study on a sample of patients treated for OSAS using CPAP. Commonly measured respiration parameters from sleep monitoring were

Table 1. Characteristics of the studied sample

\begin{tabular}{lccccc}
\hline & $\begin{array}{c}\text { Total } \\
\text { count }\end{array}$ & Male & Female & $\begin{array}{c}\text { Successful } \\
\text { CPAP }\end{array}$ & $\begin{array}{c}\text { Unsuccessful } \\
\text { CPAP }\end{array}$ \\
\hline $\begin{array}{l}\text { All treated } \\
\text { patients }\end{array}$ & 1253 & & & & \\
$\begin{array}{l}\text { Included in } \\
\text { study }\end{array}$ & 665 & 478 & 187 & 575 & 90 \\
$\begin{array}{l}\text { Seniors } \\
\text { Younger }\end{array}$ & 158 & 113 & 45 & 125 & 33 \\
\hline
\end{tabular}

compared between successfully and unsuccessfully treated patients. Additionally, the differences in respiration parameters and CPAP success rate between the older and of younger patients (i.e., above and below 65 years, respectively) were evaluated. Lastly, the ability of age and respiration parameters to predict CPAP failure was evaluated both individually and in a multivariate model.

\section{The following variables were recorded and analysed:}

- The BMI (body mass index) was defined as body weight divided by the square of height; a measure of the degree of obesity.

- The AHI (apnea-hypopnea index) was defined as the average number of apnea and hypopnea episodes per one hour of sleep.

- The ODI (oxygen distress index) was defined as the average desaturation during sleep.

- Average saturation was defined as the mean SaO2 during sleep.

- The T90 was defined by the percentage of sleep time below $90 \% \mathrm{SaO} 2$.

- Treatment failure was defined as: (A) AHI > 10 and lower than $75 \%$ decrease in baseline AHI value and (B) AHI $<10$ but persisting hyposaturation, $\mathrm{T} 90 \geq 10 \%$ and $\mathrm{ODI} \geq 10$. The effect of CPAP was assessed after 3 months of treatment.

- Inclusion criteria: (1) OSAS evaluation (diagnosis and follow-up) by the same polygraphic monitoring systems (Miniscreen 8, PolyMesam, Vitaloc, Stardust), (2) the first choice for therapy was a CPAP device, and (3) good compliance with therapy.

- Exclusion criteria: (1) previous conservative and/or surgical treatment of OSAS, (2) chronic obstructive pulmonary disease (COPD), (3) alcohol abuse, and (4) incompletely measured data.

\section{Statistical methods}

\section{Basic statistics}

Associations between CPAP failure and patient age category or gender were tested using the chi-squared test. Because of significantly non-normal distributions for most of the quantitative variables, non-parametric tests were used for subsequent analyses. Respiration parameters were compared between successfully and unsuccessfully treated patients, between genders, and between age categories using the Mann-Whitney $U$ test. Mutual correlations between quantitative variables were analysed using the Spearman's correlation coefficient. All reported p-values are two-tailed, and the level of statistical significance was set at $\alpha=0.05$.

\section{Predictive capability of individual variables}

First, the ability to predict CPAP failure from patient age category (with the empirical threshold of 65 years of age) was investigated by calculating the sensitivity and specificity for such stratification. Second, the potential of respiration parameters and age (on a continuous scale) to predict CPAP failure was assessed using the Receiver Operating Characteristic (ROC). An ROC curve was plotted for each variable and the area under the curve (AUC ROC) was then calculated to summarize the predictive strength of the variable as a single value (AUC ROC of 1 representing perfectly reliable prediction and AUC ROC of 0.5 representing random guessing). Sensitivity and specificity were determined for specific threshold values. Confidence intervals for relative frequency, sensitivity, and specificity were calculated according to Agresti and Coull (1998). 
Multivariate prediction model of CPAP failure

Based on the best-performing predictors from the single-variable analysis (T90), several multivariate models were constructed using a combination of T90 with other variables not correlated with it, e.g., BMI and age (individually). Both versions of the model (T90 + BMI and T90 + age) were then extended by including gender. The model defined a new predictive variable as a linear combination of the elementary continuous variables. This variable was then assessed in the same way as the single predictors, i.e., using the ROC analysis of a simple threshold-based stratification. For each version of the model, the linear combination coefficients were optimized to maximize the AUC ROC. The optimization was carried out using an enumerative exploration of the whole possible range of rotation angles of the combined factor with respect to the coordinate system defined by the original variables. When gender was included, separate pairs of coefficients were optimized for men and women and then combined into one model.

Basic statistical analysis was performed using STATISTICA (StatSoft, Inc. 2013, Version 12); the ROC analysis and optimization were carried out using MATLAB (Release 2014b, The MathWorks).

\section{Theory}

In our work, we focused to find patients with CPAP failure. These patients will benefit from BiPAP as the first choice for treatment. At the time, CPAP is the first choice for treatment, however, it is not always successful. In cases where CPAP was unsuccessful, treatment with BiPAP is the next treatment option. We examine commonly evaluated respiratory parameters, obesity, and age relative to their ability to predict CPAP failure (each individual parameter as well as combinations of parameters).

\section{Results}

\section{Analyses of the monitored variables and their relationship to CPAP failure}

Of the 665 patients included in the study, CPAP failure was observed in 90 cases (13.5\%; $95 \%$ confidence interval of CPAP failure rate $11.1-16.4 \%)$. All recorded pre-treatment parameters differed significantly between successfully and unsuccessfully treated patients (Fig. 1). All respiration parameters, i.e., AHI $(p=0.0002)$, T90 ( $p<0.0001)$, ODI $(p<0.0001)$, and average saturation $(p<0.0001)$ showed more serious breathing insufficiency in patients in whom CPAP failed. Similarly, CPAP failure was significantly associated with higher age ( $p=$ 0.0014) and BMI ( $p=0.0092)$.

When comparing the monitored parameters between age categories (Fig. 2a, b), older patients ( $>65$ years) had a significantly lower AHI $(p=0.0044)$ and ODI $(p=0.0038)$ compared to younger patients, while no differences were observed relative to BMI, T90, and average saturation. The same association was also confirmed on a continuum, in which age was mildly, yet significantly correlated with AHI and ODI (Table 2). The correlation analysis also showed that all the respiration-related parameters, i.e., AHI, ODI, T90, and average saturation, are significantly correlated with one another. Notably, none of the analysed variables were correlated with BMI. Concerning gender (Fig. 2c, d), male patients were significantly younger $(p=0.0002)$ and had higher T90s (0.0152) than females. The remaining patient characteristics, as well as the CPAP failure rate, did not differ significantly between genders.
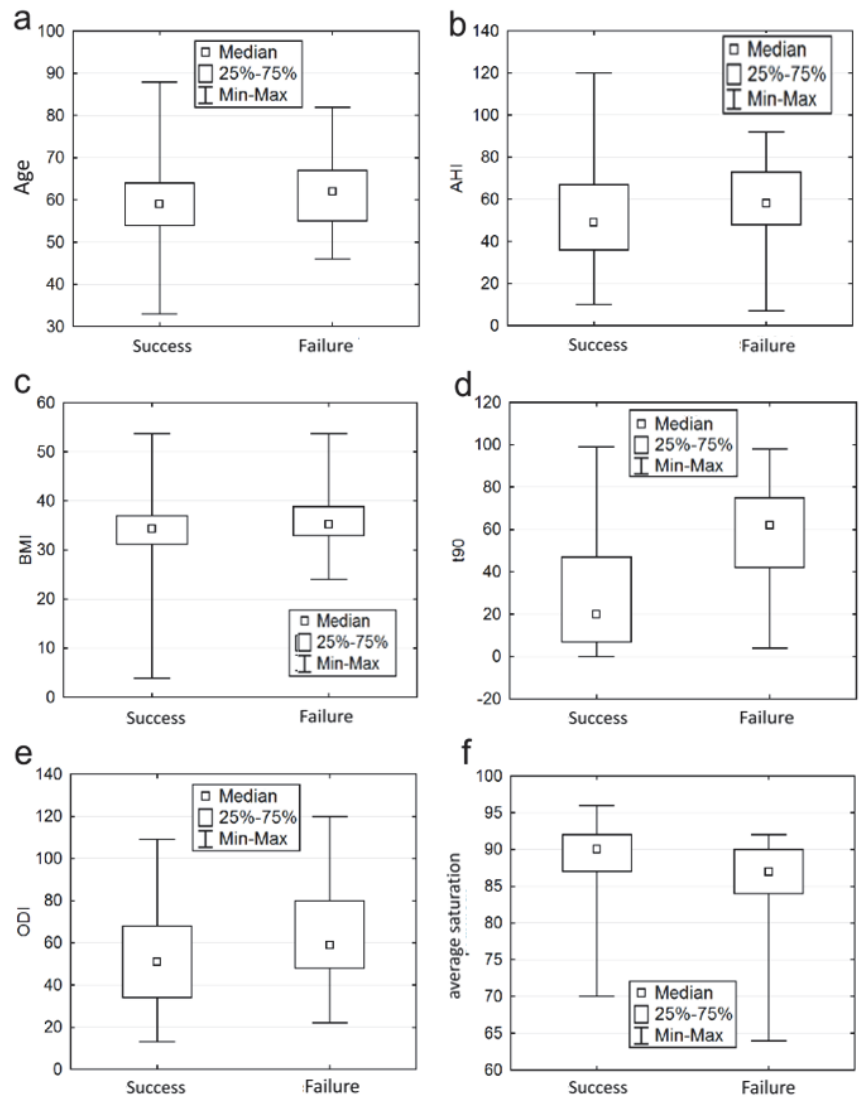

Fig. 1. Differences in the monitored variables between successfully and unsuccessfully treated patients

Note: (a) age; (b) AHI; (c) BMI; (d) T90; (e) ODI; (f) average saturation.
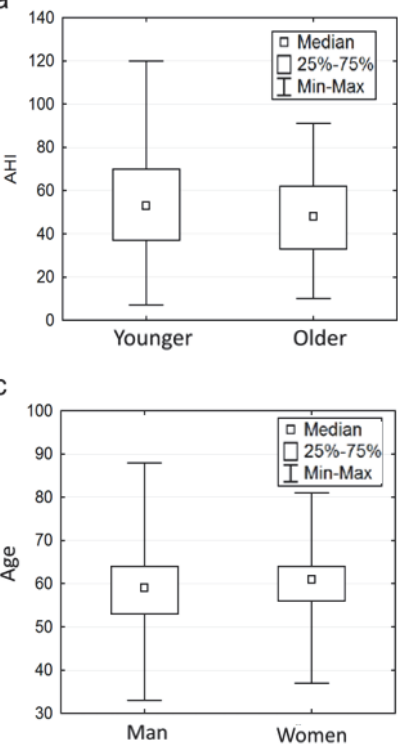

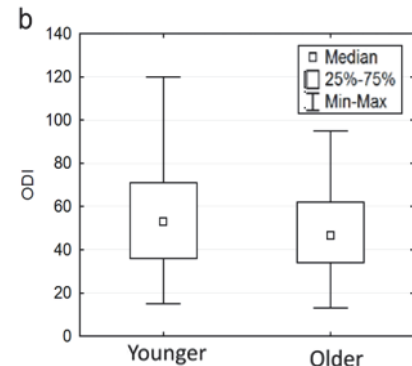

d

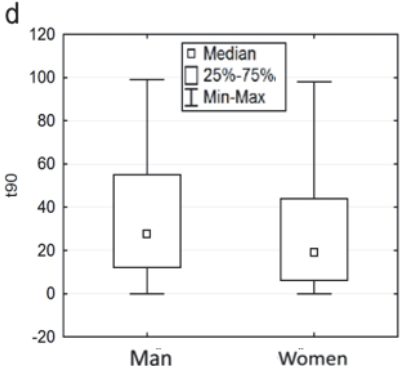

Fig. 2. Differences in the monitored variables between age categories and genders

Note: AHI (a) and ODI (b) were lower in older patients. Younger age (c) and greater T90 (d) was observed in men in comparison to women. 
Table 2. Correlations between monitored variables. Values of Spearman's correlation coefficient are shown, significant correlations are highlighted and marked in bold typeface.

\begin{tabular}{lrllllr}
\hline & \multicolumn{1}{c}{ Age } & BMI & \multicolumn{1}{c}{ AHI } & \multicolumn{1}{l}{ T90 } & \multicolumn{1}{c}{ ODI } & Avg. sat. \\
\hline Age & & 0.059 & $\mathbf{- 0 . 2 0 0}$ & 0.011 & $\mathbf{- 0 . 2 2 9}$ & -0.021 \\
BMI & 0.059 & & 0.049 & 0.033 & 0.023 & -0.049 \\
AHI & $\mathbf{- 0 . 2 0 0}$ & 0.049 & & $\mathbf{0 . 5 3 0}$ & $\mathbf{0 . 7 6 3}$ & $\mathbf{- 0 . 4 5 6}$ \\
T90 & 0.011 & 0.033 & $\mathbf{0 . 5 3 0}$ & & $\mathbf{0 . 6 4 5}$ & $\mathbf{- 0 . 8 8 8}$ \\
ODI & $\mathbf{- 0 . 2 2 9}$ & 0.023 & $\mathbf{0 . 7 6 3}$ & $\mathbf{0 . 6 4 5}$ & & $\mathbf{- 0 . 5 8 0}$ \\
Avg. sat. & -0.021 & -0.049 & $\mathbf{- 0 . 4 5 6}$ & $\mathbf{- 0 . 8 8 8}$ & $\mathbf{- 0 . 5 8 0}$ & \\
\hline
\end{tabular}

\section{Prediction of CPAP failure using individual variables}

Following the association between CPAP failure and age demonstrated above (Fig. 1a), significantly higher CPAP failure rates $(p=0.0020)$ were expectably found in older patients (20.9\%; 95\% CI 15.1-28.1) in comparison to younger patients (11.2\%; 8.7-14.4\%). When used as a single predictor of CPAP failure, the older age category showed a sensitivity of $36.7 \%$ (27.3-47.2\%) and specificity of 78.3 (74.6-81.5\%).

Next, the predictive capability of the continuous variables with respect to CPAP failure was investigated using ROC analysis (Fig. 3a-f). The best predictive performance was shown by T90 (AUC ROC 0.791), followed by average saturation (0.723). The remaining parameters performed rather poorly, decreasing in quality from ODI (AUC ROC 0.636), to AHI (0.620), to age (0.605), and lastly to BMI (0.585).

\section{Predicting CPAP failure from multiple variables}

While the combined model using T90 and BMI (AUC ROC 0.793) exhibited negligible improvement over T90 alone (0.791), the model combining T90 with age showed a meaningful improvement of prediction quality (0.812, Fig. 3g). Subsequent inclusion of gender brought further improvements to both variants, nonetheless, the model including BMI (i.e., T90 + BMI + gender, AUC ROC 0.801) was still clearly outperformed by the variant featuring age instead (i.e., T90 + age + gender, AUC ROC 0.822, Fig. 3h). The latter was also the best performing predictor constructed from our dataset, reaching almost $80 \%$ sensitivity and specificity at their point of equality.

\section{Discussion}

Sleep apnea syndrome is a disabling condition that can affect people of all ages but is particularly problematic in older patients, who are more vulnerable to adverse cardiovascular events and disabilities (Monahan and Redline, 2011). Considering that in elderly patients, there is a special emphasis put on minimally invasive and conservative procedures, a simple method for predicting successful treatment is essential.

In our study, we discussed commonly evaluated respiratory parameters, as well as obesity (measured by BMI), and age as predictive factors of CPAP therapy failure. We have tried to find differences in the predictive capabilities of these parameters in elderly and younger patients. To date, this study is, to the best of our knowledge, the largest to explore the interplay between these parameters and CPAP failure.

In our sample, $13 \%$ of patients did not achieve satisfactory results after 3 months of CPAP treatment and these patients had to be switched to BiPAP ventilation. Comparable results were published by Schäfer et al. (1998) in a smaller sample of 146 patients. Their findings found BMI and T90 to be predictors. BMI had a median value of 31 in successfully, and 44 in unsuccessfully treated patients, T90 had a median value of 15 in the successfully, and 82 in the unsuccessfully treated group (Schäfer et al., 1998).

Although BMI, AHI, ODI, T90, and SaO2 were statistically significant risk factors for CPAP failure in our work, the ROC analysis suggested that none of these parameters alone can be used for the prediction of CPAP failure with clinically relevant reliability. Their strength as individual variables would have to have been much higher, to reach the limit necessary for clinical significance.

We assumed that age would influence the success rate of CPAP treatment since impairment in cognitive abilities associated with older age would be expected to impact compliance (Šedová et al., 2016). Good communication plays a particularly critical role in elderly patients (La Piana et al., 2011; Morales et al., 2012). Our supposition was confirmed; however, the predictive ability of age was found too weak for clinical praxis. T90 proved to be a predictor of treatment outcomes in line with Schäfer's as well as our previous conclusions based on a smaller sample (Schäfer et al., 1998). When we used combined models we got pronounced increases in the predictive ability, i.e., with the T90 and age combination (AUC ROC 0.812, Fig. 3g) and even better with the T90, age, and gender combination (AUC ROC 0.822, Fig. 3h). Unfortunately, we were not able to compare our conclusions because no other studies have tried using combined models of predictive factors relative to CPAP treatment failure.

We are aware that a single center study may limit the generalization of our results, even though our sample of patients was large and unselected (representative) from a large regional University Hospital. The demographic distribution of our patients supports our findings (Monahan and Redline, 2011), nonetheless, prospective multi-center studies would be useful for continued exploration of the problem.

\section{Conclusions}

The number of patients with SAS is significant and as medical care continues to improve, the number of patients diagnosed and treated for SAS can be expected to increase. The medical and socio-economic impact of untreated or poorly treated patients is serious, and as such requires proper diagnosis and treatment. A model that combines T90, age, and gender greatly facilitates finding patients who will benefit from BiPAP as the first-choice device, and therefore, should produce relevant reductions in treatment cost associated with SAS.

\section{Ethical aspects}

The Ethics Committee of the University Hospital in Pilsen granted their approval to the data analyses.

\section{Conflict of interests}

The authors have no conflict of interests to declare. 


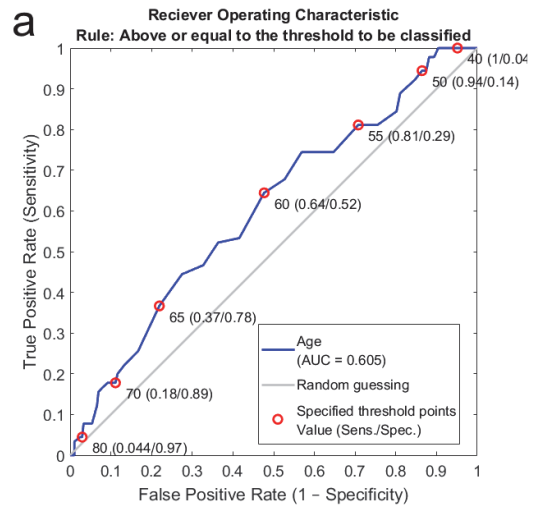

False Positive Rate (1 - Specificity)
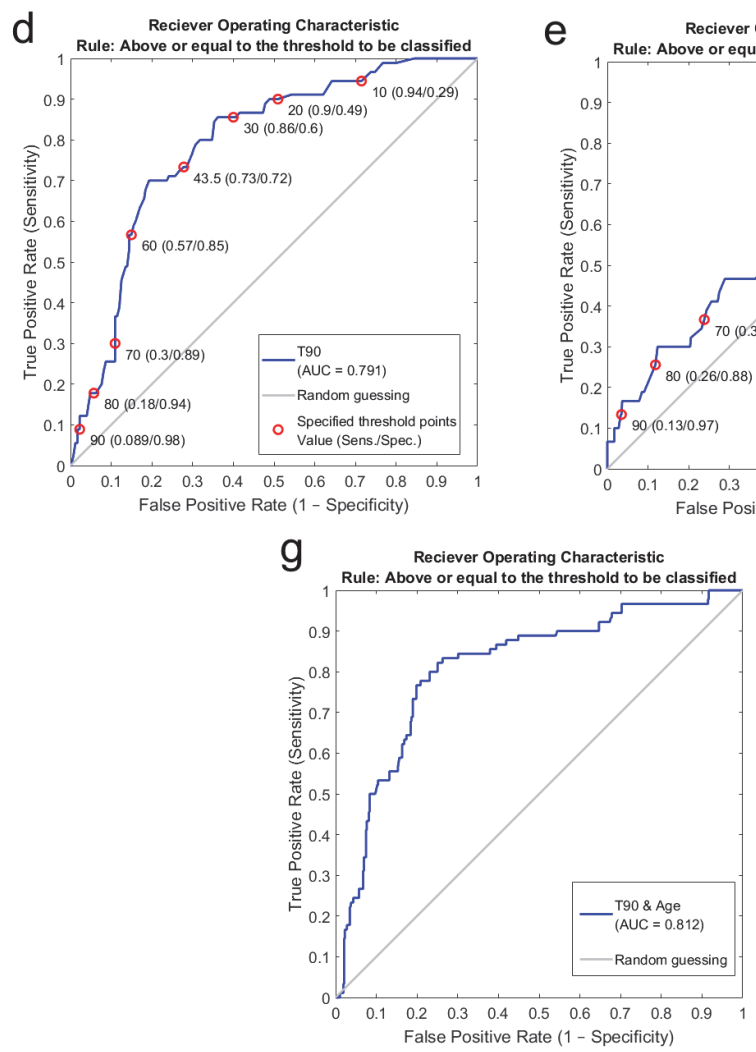

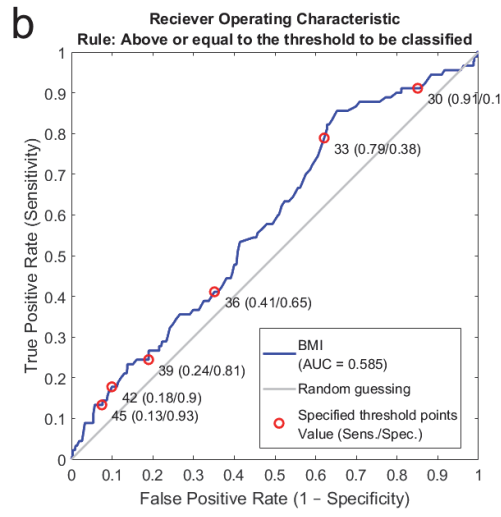

C Reciever Operating Characteristic
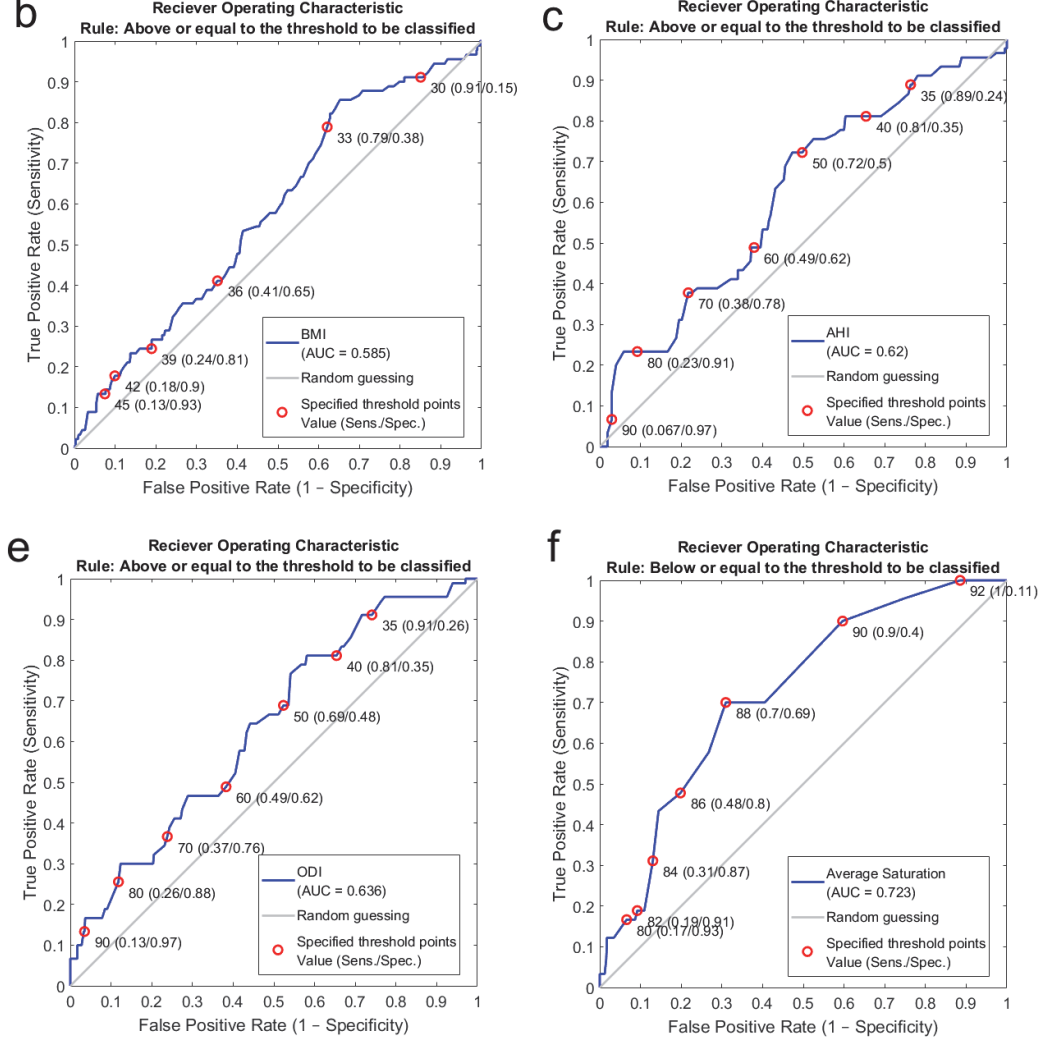

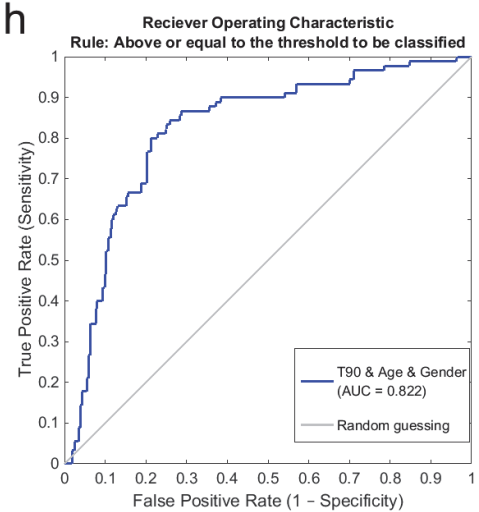

Fig. 3. ROC curves for CPAP failure prediction

Note: (a) age; (b) BMI;(c) AHI; (d) T90; (e) ODI; (f) average saturation; (g) combined model using T90 and age; (h) combined model using T90, age, and gender.

\section{Acknowledgements}

This project was supported by the grant of Ministry of Health of the Czech Republic - Conceptual Development of Research Organization (Faculty Hospital in Pilsen - FNPl, 00669806), and by the National Sustainability Program I (NPU I) No. LO1503 provided by the Ministry of Education Youth and Sports of the Czech Republic.

\section{References}

Agresti A, Coull BA (1998). Approximate is better than "exact" for interval estimation of binomial proportions. Am Statistician 52(2): 119-126. DOI: 10.2307/2685469.
Barbé F, Durán-Cantolla J, Sánchez-de-la-Torre M, MartínezAlonso M, Carmona C, Barceló A, et al. (2012). Effect of continuous positive airway pressure on the incidence of hypertension and cardiovascular events in nonsleepy patients with obstructive sleep apnea: a randomized controlled trial. JAMA 307(20): 2161-2168. DOI: 10.1001/jama.2012.4366.

Browaldh N, Markström A, Friberg D (2009). Elective tracheostomy is an alternative treatment in patients with severe obstructive sleep apnoea syndrome and CPAP failure. Acta Otolaryngol 129(10): 1121-1126. DOI: 10.1080/00016480802572509.

Bruin PF, Bagnato M (2010). Cognitive impairment in obstructive sleep apnea syndrome. J Bras Pneumol 36 (Suppl. 2): 32-37. DOI: $10.1590 /$ S1806-37132010001400010.

Butner KL, Hargnes TA, Kaleth AS (2013). Association of obstructive sleep apnea severity with exercise capacity and health-related quality of life. N Am J Med Sci 5(6): 362-366. DOI: 10.4103/19472714.114168. 
Chowdhury O, Wedderburn CJ, Duffy D, Greenough A (2012). CPAP review. Eur J Pediatr 171(10): 1441-1448. DOI: 10.1007/s00431011-1648-6.

Dohi T, Kasai T, Narui K, Ishiwata S, Ohno M, Yamaguchi T, Momomura S (2008). Bi-level positive airway pressure ventilation for treating heart failure with central sleep apnea that is unresponsive to continuous positive airway pressure. Circ J 72(7): 1100-1105. DOI: 10.1253/circj.72.1100.

Dostálová S, Susta M, Vorlová T, Šonka K (2012). Sleepiness in patients with obstructive sleep apnoea - daytime course and impact of nocturnal respiratory events. Neuro Endocrinol Lett 33(7): 684-688.

Ephros HD, Madani M, Yalamanchili SC (2010). Surgical treatment of snoring \& obstructive sleep apnoea. Indian J Med Res 131: 267-276.

Epstein LJ, Kristo D, Strollo PJ Jr, Friedman N, Malhotra A, Patil SP, et al. (2009). Clinical guideline for the evaluation, management and long-term care of obstructive sleep apnea in adults. J Clin Sleep Med 5(3): 263-276.

Jalbert F, Lacassagne L, Bessard J, Dekeister C, Paoli JR, Tiberge M (2012). Oral appliances or maxillomandibular advancement osteotomy for severe obstructive sleep apnoea in patients refusing CPAP. Rev Stomatol Chir Maxillofac 113(1): 19-26. DOI: 10.1016/j.stomax.2011.11.005.

Kasai T (2012). Sleep apnea and heart failure. J Cardiol 60(2): 78-85. DOI: 10.1016/j.jjcc.2012.05.013.

La Piana GE, Scartabellati A, Chiesa L, Ronchi L, Raimondi P, Carro M, et al. (2011). Long-term adherence to CPAP treatment in patients with obstructive sleep apnea: importance of educational program. Patient Prefer Adherence 5: 555-562. DOI: 10.2147/ PPA.S24018

Leidag M, Hader C, Keller T, Meyer Y, Rasche K (2008). Mask leakage in continuous positive airway pressure and C-Flex. J Physiol Pharmacol 59 (Suppl. 6): 401-406.

Mannarino MR, Di Filippo F, Pirro M (2012). Obstructive sleep apnea syndrome. Eur J Intern Med 23(7): 586-593. DOI: 10.1016/j.ejim.2012.05.013.

Maurer JT (2010). Surgical treatment of obstructive sleep apnea: standard and emerging techniques. Curr Opin Pulm Med 16(6): 552-558. DOI: 10.1097/MCP.0b013e32833ef7ea.

Monahan K, Redline S (2011). Role of obstructive sleep apnea in cardiovascular disease. Curr Opin Cardiol 26(6): 541-547. DOI: 10.1097/HCO.0b013e32834b806a.

Morales CR, Hurley S, Wick LC, Staley B, Pack FM, Gooneratne NS (2012). In-home, self-assembled sleep studies are useful in diagnosing sleep apnea in the elderly. Sleep 35(11): 1491-1501. DOI: 10.5665/sleep.2196.
Mulgrew AT, Lawati NA, Ayas NT, Fox N, Hamilton P, Cortes L, Ryan CF (2010). Residual sleep apnea on polysomnography after 3 months of CPAP therapy: clinical implications, predictors and patterns. Sleep Med 11(2): 119-125. DOI: 10.1016/j. sleep.2009.05.017.

Onen F, Onen H (2010). Obstructive sleep apnea and cognitive impairment in the elderly. Psychol Neuropsychiatr Vieil 8(3): 163-169. DOI: 10.1684/pnv.2010.0219.

Piper AJ, Wang D, Yee BJ, Barnes DJ, Grunstein RR (2008). Randomised trial of CPAP vs bilevel support in the treatment of obesity hypoventilation syndrome without severe nocturnal desaturation. Thorax 63(5): 395-401. DOI: 10.1136/ thx.2007.081315.

Schäfer H, Ewig S, Hasper E, Lüderitz B (1998). Failure of CPAP therapy in obstructive sleep apnoea syndrome: predictive factors and treatment with bilevel-positive airway pressure. Respir Med 92(2): 208-215. DOI: 10.1016/S0954-6111(98)90097-X.

Šedová L, Doskočil O, Brabcová I, Hajduchová H, Bártlová S (2016). Selected aspects of health literacy among seniors. Neuro Endocrinol Lett 37(Suppl. 2): 11-17.

Sopkova Z, Dorkova Z, Tkacova R (2009). Predictors of compliance with continuous positive airway pressure treatment in patients with obstructive sleep apnea and metabolic syndrome. Wien Klin Wochenschr 121(11-12): 398-404. DOI: 10.1007/s00508-0091181-z.

Tóthová V, Bártlová S, Šedová L, Olišarová V, Prokešová R, Adámkova V, et al. (2015). The importance of self-management in the prevention and treatment of excessive weight and obesity. Neuro Endocrinol Lett 36 (Suppl. 2): 5-10.

Tureto-Borges F, Jenner B, Costa-Hong V, Lopes HF, Teixeira SH, Marum E, et al. (2018). Does obstructive sleep apnea influence blood pressure and arterial stiffness in response to antihypertensive treatment? Hypertension 120(4): 615-627. DOI: 10.1161/HYPERTENSIONAHA.118.10825.

Vozoris NT (2012). Sleep apnea-plus: prevalence, risk factors, and association with cardiovascular diseases using United States population-level data. Sleep Med 13(6): 637-644. DOI: 10.1016/j. sleep.2012.01.004.

Williams B, Boyle M, Robertson N, Giddings C (2013). When pressure is positive: a literature review of the prehospital use of continuous positive airway pressure. Prehosp Disaster Med 28(1): 52-60. DOI: 10.1017/S1049023X12001562. 